In der Rubrik „Literatur kompakt" werden die wichtigsten Originalarbeiten aus der internationalen Fachliteratur referiert.

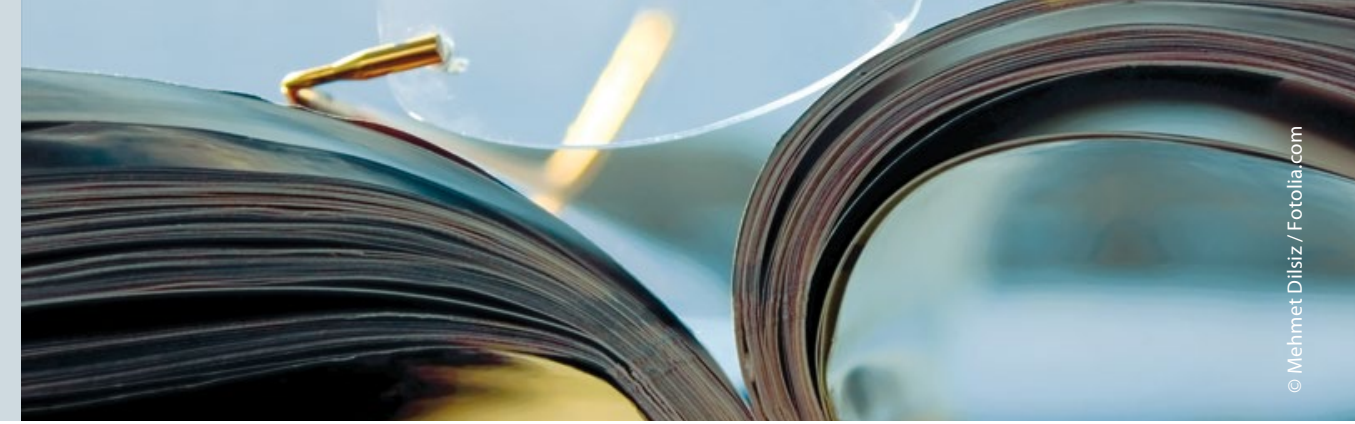

\section{Nierensteine: Für die Analyse reicht schon Nachturin}

\begin{abstract}
Urinsammeln ist für Nierensteinpatienten sehr lästig - vor allem tagsüber. Doch offenbar genügt es, zur Analyse auf den Nachturin zu setzen, haben Forscher nun analysiert.
\end{abstract}

$\mathrm{N}$ icht selten wird von Patienten mit Nierensteinen zur Analyse der 24-Stunden-Urin angefordert, vor allem dann, wenn diese Patienten unter Rezidiven leiden. Studien haben ergeben, dass eine Wiederholung des Tests wesentlich bessere Resultate liefert als eine einmalige Bestimmung des 24-StundenUrins, berichten Urologen um Dr. Brian Hinck von der Cleveland Clinic, Ohio, USA.

Für die Patienten ist es jedoch alles andere als angenehm, wenn sie ein- bis zweimal einen ganzen Tag über ihren Urin auffangen müssen. Wer beispielsweise nicht gerade im höheren Alter, in Rente oder aus anderen Gründen unter der Woche nicht arbeitstätig ist, wird diese Aufgabe daher aufs Wochenende verlagern. An den Wochenenden sind jedoch Ernährungs- und Lebensbedingen mitunter deutlich anders als unter der Woche, was die Resultate verfälschen kann, vermuten die US-Urologen. Besser wäre demnach eine Methode, die es den Patienten erlaubt, ihren Urin auch unter der Woche ohne Probleme zu sammeln.

Die Forscher um Hinck haben in einer prospektiven Studie untersucht, ob vielleicht der Nachturin genügen würde. Da die meisten Patienten nachts zu Hause sind, sollte das Urinsammeln somit auch wochentags keine Probleme bereiten.
Zudem ist der Urin nachts in der Regel konzentrierter als tagsüber, dies könnte die Aussagekraft steigern.

An der Studie der amerikanischen Forscher haben 35 Patienten mit Urolithiasis teilgenommen. Bei jedem Patienten wurde zweimal eine 24-StundenKollektion angefordert. Eine dieser Kollektionen sollte wochentags, die andere am Wochenende erfolgen. Die Patienten wurden instruiert, den Tagurin beginnend mit der zweiten Blasenentleerung nach dem Aufstehen - vom Nachturin zu separieren.

Die ersten zwölf Stunden nach dem Aufstehen flossen in Behälter für den Tagurin, die übrigen inklusive des ersten Urins nach dem Aufstehen in die Gefäße für den Nachturin. Analysiert wurden etwa Kalzium, Oxalat, Zitrat, Harnsäure und Kreatinin. Das Alter der Teilnehmer betrug im Mittel 59 Jahre, der Body-Mass-Index lag bei 31.

Die Patienten lieferten insgesamt 49 komplette Proben ab. Dabei zeigte sich eine deutliche Korrelation zwischen 24-Stunden- und Nachturinwerten. Der Korrelationskoeffizient lag zwischen 0,76 für den $\mathrm{pH}$-Wert und 0,96 für $\mathrm{Zi}$ trat. Werte von 1 kennzeichnen eine vollständige Übereinstimmung.

Bei fünf Proben fanden die US-Forscher um Hinck eine Kalziumoxalatübersättigung im 24-Stunden-Urin, die- se war auch in allen fünf zugehörigen Nachturinproben nachweisbar und zusätzlich in zwei weiteren Nachtproben. Acht 24-Stunden-Proben zeigten erhöhte Kalziumphosphatwerte, in sieben der entsprechenden Nachtproben waren die Werte ebenfalls erhöht. Neun Ganztagsproben offenbarten erhöhte Harnsäurewerte, diese zeigten sich auch in acht der Nachtproben sowie einer zusätzlichen Nachtprobe, bei der die 24-StundenWerte unauffällig waren.

Die Korrelation zwischen Tag- und Ganztagswerten war deutlich schwächer, schreiben Hinck und seine Kollegen. Unterschiede zwischen Werktagen und Wochenenden konnten praktisch keine festgestellt werden.

Fazit: Insgesamt korrelieren also vor allem die Nachtwerte mit den 24-Stunden-Werten. Da nachts häufig höhere Konzentrationen von Nierensteinbestandteilen festgestellt wurden als bei den 24-Stunden-Proben der Patienten, könnte die Analyse des Nachturins eine deutlich höhere Sensitivität für metabolische Probleme aufweisen, geben die Autoren der Studie zu bedenken. Wurden Therapieempfehlungen von den Messungen abgeleitet, stimmten diese bei der Gesamtprobe und der Nachtprobe zu $92 \%$ überein. Insgesamt würden bei der Gesamtprobe kritische Werte tendenziell eher übersehen. Dies müsse jetzt natürlich in größeren Studien evaluiert werden, schreiben Hinck und Kollegen.

Thomas Müller

Hinck BD, Ganesan V, Tarplin S et al. Can a simplified 12-hour nighttime urine collection predict urinary stone risk? Urology. 2017; https://doi. org/10.1016/j.urology.2017.06.028. 